\title{
Genetic Control of Radical Crosslinking in a Semi-Synthetic Hydrogel
}

Austin J. Graham ${ }^{1,2}$, Christopher M. Dundas ${ }^{1,2}$, Alexander Hillsley ${ }^{1}$, Dain S. Kasprak ${ }^{3}$, Adrianne M. Rosales ${ }^{1,2}$, Benjamin K. Keitz ${ }^{1,2}$

${ }^{1}$ McKetta Department of Chemical Engineering, University of Texas at Austin, Austin, TX 78712, USA

${ }^{2}$ Center for Dynamics and Control of Materials, University of Texas at Austin, Austin, TX 78712, USA

${ }^{3}$ Department of Biomedical Engineering, University of Texas at Austin, Austin, TX 78712, USA

1

\section{Abstract:}

Enhancing materials with the qualities of living systems, including sensing, computation, and adaptation, is an important challenge in designing next-generation technologies. Living materials seek to address this challenge by incorporating live cells as actuating components that control material function. For abiotic materials, this requires new methods that couple genetic and metabolic processes to material properties. Toward this goal, we demonstrate that extracellular electron transfer (EET) from Shewanella oneidensis can be leveraged to control radical crosslinking of a methacrylate-functionalized hyaluronic acid hydrogel. Crosslinking rates and hydrogel mechanics, specifically storage modulus, were dependent on a variety of chemical and biological factors, including $S$. oneidensis genotype. Bacteria remained viable and metabolically active in the crosslinked network for a least one week, while cell tracking revealed that EET genes also encode control over hydrogel microstructure. Moreover, construction of an inducible gene circuit allowed transcriptional control of storage modulus and crosslinking rate via the tailored expression of a key electron transfer protein, MtrC. Finally, we quantitatively modeled dependence of hydrogel stiffness on steady-state gene expression, and generalized this result by demonstrating the strong relationship between relative gene expression and material properties. This general mechanism for radical crosslinking provides a foundation for programming the form and function of synthetic materials through genetic control over extracellular electron transfer.

\section{Significance Statement:}

Next-generation materials will require coupling the advantages of engineered and natural systems to solve complex challenges in energy, health, and the environment. Living cells, such as bacteria, naturally possess many of the qualities essential to addressing these challenges, including sensing, computation, and actuation, using their genetic and metabolic machinery. In addition, bacteria are attractive for incorporation into materials due to their durability, ease-of-use, and programmability. Here, we develop a platform for controlling hydrogel properties (e.g., stiffness, crosslinking rate) using extracellular electron transfer from the bacterium Shewanella oneidensis. In our system, metabolic electron flux from $S$. oneidensis to a metal catalyst generates radical species that crosslink an acrylate-based macromer to form the gel. This synthetic reaction 
30 is under direct control of bacterial genetics and metabolism, which we demonstrate through

31 inducible circuits and quantitative modeling of gene expression and resultant hydrogel properties.

32 Developing methods that capitalize on the programmability of biological systems to control

33 synthetic material properties will enable hybrid material designs with unprecedented functions.

34 Introduction:

Nature uses hierarchical and genetically-encoded instructions to construct functional materials with specific self-assembly, regulatory, healing, and morphological properties(1). Inspired by such processes, engineered living materials (ELMs) employ the autonomy of living cells to synthesize and control material structures across multiple scales with user-designed functions that are directly coupled to gene expression(2-5). Living materials containing microbes, including biofilms, bacterial cellulose, curli fibers, and synthetic gels loaded with bacteria, are of prominent interest due to their potential application in tissue engineering, 3D printing, soft robotics, metabolic engineering, and living sensors(6-10). Bacteria are particularly attractive as ELM components due to their natural sensing capabilities and programmability. For example, engineered bacteria can act as cellular actuators integrated within the ELM, tailoring its synthesis and function through overexpression, mutagenesis, and gene circuitry.

Not surprisingly, the majority of ELMs rely on materials natively produced by the host organism. For example, several amyloid-based materials have been synthesized by genetically tractable bacteria, such as aggregates of CsgA in Escherichia coli $(11,12)$ and TasA in Bacillus subtilis(13). Genetic fusions have allowed these fibrous matrices to bind specific molecules, conduct electricity, perform catalytic reactions, and adhere to complex surfaces(14-17). Apart from amyloids, extracellular polymerization of bacterial cellulose has been engineered using quorum sensing circuits and mutagenesis to create sturdy materials for tissue engineering and sensing applications $(18,19)$. Despite these advances, significant drawbacks of natural materials

54 include their limited chemical functionality, robustness, homogeneity, and scalability compared to 55 engineered synthetic materials(20). For example, manufactured soft materials such as polymers 56 and hydrogels are easily-functionalized and versatile, facilitating their adoption in diverse 57 environments. However, synthetic materials largely lack the dynamic adaptability and 58 environmental responsiveness found in natural systems. Introducing these qualities to synthetic 59 materials could synergistically enhance ELMs and enable new applications that combine the 60 precision and chemical diversity of engineered materials with the autonomy and evolvability of 61 living cells. However, such designs will require methods for bacteria to control synthetic material 62 properties at the genotypic level. Similarly, robust transcriptional control and quantitative 
63 prediction of the relationship between gene expression and material properties are needed for

64 ELMs to approach the design precision of engineered materials.

65 Toward this goal, we recently developed a cell-controlled radical polymerization reaction 66 using extracellular electron transfer (EET) from the organism Shewanella oneidensis(21). In this 67 process, electron flux from native carbon metabolism was redirected to a metal catalyst which 68 controlled a polymerization governed by the atom-transfer radical polymerization (ATRP) 69 mechanism. Importantly, we demonstrated that control over polymer production was directly 70 coupled to cell metabolism and genetically encoded through specific EET proteins. Since ATRP 71 is a versatile platform for soft material synthesis(22), we hypothesized that EET-powered catalysis 72 could be extended to control radical crosslinking in a synthetic hydrogel. While there are 73 numerous examples of incorporating live cells into polymer networks, network properties such as 74 crosslink density, mesh size, degradation, and elastic modulus have generally been designed 75 independent of cell activity. In addition, previous attempts to incorporate cells as live crosslinking 76 agents in synthetic hydrogels have relied on the activity of glucose peroxidase or extracellular functionalization of cells after growth(23-27), which compromise cell viability through the creation of toxic reactive oxygen species or are not under cellular control. Cell-free gelation systems using bacterial lysates have also been explored(28), but removing the living component prevents continued responsiveness. We envisioned that controlling radical crosslinking via EET gene expression would capitalize on the programmability of bacteria and enable the use of stimuliresponsive synthetic biology circuits to control material function.

Here, we demonstrate that EET from $S$. oneidensis can be used to control radical 84 crosslinking of a semi-synthetic methacrylated hyaluronic acid (MeHA) hydrogel (Fig. 1a). First, 85 we show that EET is required for gelation, and that organisms without this metabolic capability 86 (i.e., E. coli) are unable to crosslink gels on a comparable time scale or in a controllable manner. 87 Gels did not form unless a constant source of electron flux, radical initiator, and metal catalyst 88 were present. Additionally, the facultative metabolic capability of $S$. oneidensis enabled 89 crosslinking under benchtop conditions without dedicated oxygen removal. Analysis of cell motility 90 and metabolic activity reveled that bacteria remain viable and responsive in the gels for a 91 minimum of one week, and that degree of crosslinking by EET affected cell movement. Next, we 92 found that crosslink density was a strong function of bacterial genetics, as cytochrome knockout 93 strains synthesized gels more slowly and with decreasing stiffness correspondent to the number 94 of removed EET genes. Finally, transcriptional circuits based on controlling the expression of $95 \mathrm{mtrC}$ with the Lacl repressor enabled tunable crosslinking rates and hydrogel mechanical 96 properties. We found that hydrogel storage modulus fit well to inducible gene expression models, 
97 directly linking steady-state gene expression to a quantifiable and macroscopic material property.

98 Overall, our results suggest that transcriptional control over EET can be used to predictably 99 interface the properties of living systems with potentially any material amenable to radical 100 crosslinking.

101 Results:

102 Extracellular Electron Transfer from Live S. oneidensis Controls Aerobic Radical 103 Crosslinking

104 To initially validate our hypothesis that EET-controlled ATRP could be used to form a 105 crosslinked hydrogel, we first synthesized a 65\% methacrylated hyaluronic acid (MeHA) 106 macromer using an established protocol(29) (Fig. S1). Hyaluronic acid is a common naturally107 derived biomaterial platform that is attractive for our application due to its biocompatibility and 108 chemical versatility(30). The high density of functional groups was chosen to increase likelihood 109 of successful crosslinking and to minimize the effect of radical scavenging by oxygen. In initial 110 experiments, MeHA was dissolved at 3 wt.\% in Shewanella Basal Medium (SBM) supplemented 111 with casamino acids (Table S2,3), and the dissolved macromer was mixed with a radical initiator, 112 2-hydroxyethyl 2-bromoisobutyrate (HEBIB, $500 \mu \mathrm{M})$, a copper catalyst with Tris(2113 pyridylmethyl)amine ligand (Cu-TPMA, $10 \mu \mathrm{M})$, and inoculated with anaerobically pregrown $S$. 114 oneidensis MR-1 cells $\left(\mathrm{OD}_{600}=0.2\right)$. Lactate $(20 \mathrm{mM})$ was the electron donor and fumarate (40 $115 \mathrm{mM}$ ) was the primary electron acceptor. After mixing, the solution was placed in a humidified 116 anaerobic chamber and was monitored via inversion testing. After $2 \mathrm{~h}$, solutions containing $S$. 117 oneidensis crosslinked to form polymer networks, whereas solutions containing $E$. coli did not 118 (Fig. 1b). Consistent with our previous results(21), these data suggest that electron flux to the 119 metal catalyst from EET-based metabolism is required for radical generation and crosslinking.

120 Taking advantage of its facultative metabolism, we next tested radical crosslinking of 121 hydrogels by $S$. oneidensis under ambient as opposed to anaerobic conditions. Using the same 122 reagent concentrations as above with aerobically pregrown cells (henceforth, standard 123 conditions), S. oneidensis formed crosslinked networks at $30^{\circ} \mathrm{C}$ in microcentrifuge tubes without 124 dedicated oxygen removal, as confirmed via inversion test. After these preliminary 125 demonstrations, we more thoroughly investigated the mechanical properties of the gels using 126 shear oscillatory rheology. $50 \mu \mathrm{L}$ solutions were inoculated and placed between two 127 hydrophobically-treated glass slides with a $0.5 \mathrm{~mm}$ silicone spacer, allowed to crosslink, and 128 swollen overnight at room temperature in 1x PBS. Storage and loss moduli were determined from 129 the linear viscoelastic regime as determined by strain and frequency sweeps (Fig. S2), and 130 calculated using a 0.01 to $100 \mathrm{~Hz}$ frequency sweep at $0.1 \%$ strain. Gels formed in both aerobic 
and anaerobic environments yielded comparable mechanical properties and were predominantly elastic networks (Fig. S3). Gels prepared by S. oneidensis were also mechanically similar to acellular gels crosslinked using UV light and the photoinitiator lithium phenyl-2,4,6trimethylbenzoylphosphinate (LAP, $500 \mu \mathrm{M}$ ) (Fig. S4). In addition, controls lacking (a) EET-active bacteria, (b) radical initiator, (c) metal catalyst, or (d) methacrylate functional group did not form measurable gels within $2 \mathrm{~h}$ (Fig. 2a, Fig. S5). Together, these results demonstrate that EETcrosslinked hydrogels can be synthesized under ambient or anaerobic conditions using electroactive bacteria to form mechanically robust networks that are typical of this macromer.

The crosslinking kinetics and mechanical properties of polymer networks strongly depend on a variety of chemical factors such as catalyst and ligand identity, rate of initiation, and initiator structure(31). Thus, we next explored the tunable range of hydrogel stiffness after $2 \mathrm{~h}$ of crosslinking by altering the concentration and identity of the chemical components in our system (Fig. S6). First, we varied the concentration of the metal catalyst from $5 \mu \mathrm{M}$ to $20 \mu \mathrm{M}$. Greater catalyst concentrations were not considered due to copper-induced transcriptional responses in Shewanella at concentrations greater than $20 \mu \mathrm{M}(32)$. Increasing catalyst concentration correspondingly increased gel modulus (Fig. 2b). Second, we varied the concentration of the initiator over the range of 250 to $1000 \mu \mathrm{M}$. As expected, increasing gel stiffness was a function of increasing initiator concentration (Fig. 2c). An additional advantage of ATRP over hydroxyl radicals is the potential for using structurally well-defined radical initiators. Consistent with this expectation, we found that a PEG-based initiator, poly(ethylene glycol) bis(2-bromoisobutyrate)

$151\left(\mathrm{M}_{\mathrm{n}, \text { avg }}=700 \mathrm{~g} / \mathrm{mol}\right)$, also successfully crosslinked EET-controlled gels at a variety of 152 concentrations (Fig. S7). Overall, EET-controlled hydrogels exhibited a modulus range of about

153 1-6 kPa for the conditions tested, which is typical of chain-growth crosslinked MeHA hydrogels 154 and within range for a variety of applications, such as biofilm and tissue mimetics $(29,33,34)$.

155 These results also indicate that traditional approaches to tuning hydrogel mechanics are still 156 applicable when using EET-controlled crosslinking.

Next, we investigated the role of inoculating S. oneidensis cell density, and thus aggregate

158 EET flux, on hydrogel modulus. Below a certain critical inoculum (around $\mathrm{OD}_{600}=0.1$ ), the rate of 159 bacterial oxygen consumption was not fast enough to overcome oxygen diffusion and radical 160 quenching. As expected, hydrogels formed with sufficient cell density, and stiffness strongly 161 correlated with $\mathrm{OD}_{600}$ (Fig. 2d). Based on these results, we predicted that crosslinking rate would 162 also be coupled to EET and initial cell density. To confirm this, we performed rheological 163 measurements in situ, which provided real-time measurement of mechanical properties during 164 crosslinking, at $1 \mathrm{~Hz}$ and $0.1 \%$ strain. Gels formed at higher initial cell concentrations were not 
165 only stronger, but formed more quickly (Fig. 2e). Consistent with end-point experiments, in situ 166 rheology measurements also confirmed that a critical concentration of cells was necessary for 167 oxygen depletion. Together, these results demonstrate that cells play a direct role in crosslinking, 168 and overall stiffness and crosslinking rate can be controlled by cell inoculum. They also suggest 169 that genetic and metabolic manipulations to tune EET flux could be used to influence gel 170 mechanics.

\section{S. oneidensis Remain Viable and Metabolically Active in the Polymer Network}

For a living material to maintain responsiveness, it is critical that the actuating components (i.e., cells) remain viable and encased in the network. The various components of our system, including the $\mathrm{Cu}$ catalyst, initiator, and presence of radicals could affect cell viability. Thus, we assessed cell viability and activity after crosslinking. EET-crosslinked gels formed in standard conditions were swollen overnight in 1x PBS after modulus measurements, and stained using BacLight Live/Dead dyes. Even after mechanical stresses induced by swelling and rheometer measurements, cells maintained approximately $100 \%$ viability 5 days after crosslinking (Fig. S9). In addition, cells exposed to crosslinking conditions, but released from the gel surface during swelling, could successfully inoculate new cultures in fresh growth media, indicating viability; these cultures were also able to crosslink new hydrogels with identical properties (Fig. S10). quantified escape or leakage of bacteria from the gels after crosslinking. At the functional group density and crosslink molecular weight of our material, the mesh size of a fully converted gel should be on the order of $10-50 \mathrm{~nm}(35,36)$. Because this is considerably smaller than average bacterial dimensions, there should be minimal cell escape. To test this prediction, crosslinked gels were prepared at standard conditions, swollen in $1 \mathrm{~mL}$ of $1 \mathrm{x}$ PBS, and the optical density of the surrounding media was measured. An initial, low optical density of cells was detected immediately upon swelling. We hypothesized that this was due to an instantaneous egress of cells on the periphery of the gels and not contained in the network. After washing gels $3 x$ with 1 $\mathrm{mL}$ PBS to remove this outer layer of cells, no increase in optical density was detected.

192 Furthermore, colony counting confirmed that escaped cells after $24 \mathrm{~h}$ of swelling accounted for < $1930.005 \%$ of the inoculating density (Fig. S11), suggesting embedded cells do not escape the 194 network in significant numbers.

Continued network adaptation and design of new functions requires an understanding of 196 spatiotemporal cell behavior within the gels during synthesis. Therefore, we next visualized the 197 relationship between genotype, crosslink density, and cell movement during gelation. We 198 constructed an inducible sfgfp expression plasmid under the control of the Lacl repressor protein 
and its cognate promoter, $P_{\text {tac }}$. Cells were transformed with this vector such that sensing of isopropyl ß-D-1-thiogalactopyranoside (IPTG) would induce a fluorescent response indicative of metabolic activity. We developed two reporter strains by transforming both $S$. oneidensis MR-1 and $\Delta m \operatorname{trC} \Delta o m c A \Delta m t r F$ (an EET-deficient knockout, described below) with this construct. Strains were grown overnight in $1000 \mu \mathrm{M}$ IPTG, washed, and inoculated into standard gelation mixtures. The solution was then pipetted onto a glass slide and sealed under a coverslip, such that crosslinking occurred in the sealed layer. Bacterial movement was monitored by time-lapse imaging using GFP fluorescence. Cells were uniformly dispersed within the network throughout gelation. For both $S$. oneidensis MR-1 and $\Delta m \operatorname{trC} \Delta o m c A \Delta m t r F$, a significant degree of bacterial motion was visible upon inoculation, both by convective flow of the reaction mixture and by flagella-based swimming(37). Minutes after inoculation, cell movement and bulk fluid motion was arrested in the S. oneidensis MR-1 sample as crosslinking proceeded (Movie S1). Contrastingly, movement both from flow and swimming were still perceptible after $2 \mathrm{~h}$ in the $\Delta m \operatorname{tr} \Delta \Delta \mathrm{omcA \Delta mtrF}$ sample, indicating that minimal crosslinking occurred (Movie S2). Cell movement was quantified using TrackMate in Fiji 1.0(38), which revealed that average cell displacement over 5 seconds was significantly greater for the knockout strain at both 0 and $2 \mathrm{~h}$ (Fig. 3a, Fig. S12). Movement was not significantly different between the two strains in non-functionalized hyaluronic acid solution, suggesting the observed motility differences were due to crosslinking, even at early times immediately following inoculation (Fig. S13, Movies S3-4). These results confirm that cells become trapped in the polymer network as it forms, and suggest that bacterial genotype encodes control over bulk and microscopic properties such as crosslink density and mesh size, affecting flow, diffusion, and cell movement within the material.

Although the cells remained viable for days in the crosslinked gels, we wished to assess their continued sensing and metabolic capabilities over long periods after crosslinking. Gels synthesized at standard conditions with sfGFP-expressing S. oneidensis MR-1 were swollen in 1x PBS for varying lengths of time after crosslinking, then induced for $24 \mathrm{~h}$ with $1000 \mu \mathrm{M}$ IPTG. Significant fluorescence was detected by microscopy in induced samples up to 1 week after crosslinking (Fig. S14), but was not detectable in uninduced samples. Together, these results indicate that the bacteria remain viable, trapped, and maintain transcriptional and translational capabilities for extended periods after crosslinking.

\section{Bacterial Genetics Govern Crosslink Density}

Understanding the genetic link between EET and crosslinking is critical for biologically controlling hydrogel structure and function. Toward this goal, we employed various EET knockout strains in crosslinking reactions. The Mtr pathway is a primary source of EET flux in anaerobic $S$. 
oneidensis metabolism (Fig. 1a). Outer membrane cytochromes MtrC and OmcA are terminal reductases of the Mtr pathway, and responsible for direct transfer of electrons onto metal species such as $\mathrm{Fe}$ and $\mathrm{Cu}(21,39)$. MtrF is a homologue to MtrC and can similarly reduce a variety of metals(40). We employed three different cytochrome knockout strains in assessing the role of EET in crosslinking: $\Delta m \operatorname{trC} \Delta o m c A, \Delta m t r C \Delta o m c A \Delta m t r F$, and $\Delta \mathrm{Mtr}$. The knockout $\Delta \mathrm{Mtr}$ refers to a strain with a large number of EET genes knocked out that should provide minimal electron flux to the catalyst (Table S1)(39, 41). E. coli MG1655 was also included as an EET-deficient control. We measured in situ crosslinking kinetics using these strains, and compared crosslinking rates and density. Both crosslinking rate and hydrogel storage modulus strongly corresponded with

242 bacterial genotype, where decreasing number of EET genes led to decreased crosslinking rates and weaker moduli (Fig. $3 \mathrm{~b}$ ). Although MtrC is the primary terminal reductase for many metal substrates, our results show that MtrF exhibits compensatory reduction of $\mathrm{Cu}$ in the $\Delta m t r C \Delta o m c A$ knockout compared to the $\Delta m t r C \Delta o m c A \Delta m t r F$ knockout. The strong similarity between gels formed by the $\Delta m \operatorname{tr} C \Delta o m c A \Delta m t r F$ and $\Delta$ Mtr knockouts further demonstrates that outer membrane cytochromes are primarily responsible for electron transfer to the $\mathrm{Cu}$ catalyst and subsequent crosslinking activity. The minimal, delayed crosslinking activity of $E$. coli suggests that background radical generation or non-specific $\mathrm{Cu}$ reduction can produce weak gels at extended times. In separate experiments, we corroborated these in situ results using end-point, swollen gel measurements after 1 and $2 \mathrm{~h}$ of crosslinking (Fig. 3c, Fig. S15). S. oneidensis MR-1 and $\Delta m t r C \Delta o m c A$ formed gels the fastest and were measurable at $1 \mathrm{~h}$, whereas the other strains did not form measureable gels by this time. Measurable networks were formed by $\Delta m \operatorname{tr} \Delta \mathrm{O}$ mcA $\Delta m t r F$ and $\Delta \mathrm{Mtr}$ at $2 \mathrm{~h}$, but were significantly weaker than gels formed by the strains containing more EET machinery. Overall, these results show that bacterial genotype directly governs gel modulus and suggests that material properties can be controlled through more sophisticated regulation of 257 EET.

258 Transcriptional Regulation of Extracellular Electron Transfer Yields Tunable Crosslinking Activity

For ELMs to emulate the adaptability of biological materials, the actuating components should continually sense and respond to their environment. Environmental stimuli should then

262 induce a transcriptional response and impart control over material properties. Toward this goal, 263 we constructed an inducible $m t r C$ expression plasmid using the same genetic circuit outlined 264 before, but replacing sfgfp with $m \operatorname{trC}$ (Fig. S16-17). We transformed the $\Delta m \operatorname{trC} \Delta$ omcA $\Delta m \operatorname{tr} F$ strain 265 with this plasmid, such that IPTG would sequentially activate $m t r C$ expression, electron transfer, 266 and crosslinking activity. Upstream of the $m t r C$ gene, a computationally-predicted weak synthetic 
ribosome binding site was employed to optimize control over EET and minimize leaky expression(42). SDS-PAGE and heme staining of total protein from induced and uninduced cell lysates validated inducible MtrC protein production after overnight growth in IPTG-containing medium. High molecular weight bands corresponding to the size of the MtrCAB complex were observed in induced $\triangle m t r C \Delta O m c A \Delta m t r F$ samples and a wild-type control, but not in uninduced and empty vector $\Delta m t r C \Delta o m c A \Delta m t r F$ samples (Fig. S18). Functional steady-state expression of $m t r C$ in response to IPTG was further validated by measuring $\mathrm{Fe}^{3+}$ reduction with the ferrozine assay. After $2 \mathrm{~h}$ of reduction, $\mathrm{Fe}^{2+}$ concentration increased with the presence of inducing molecule, indicating functional MtrC activity and no leaky EET response over an uninduced control (Fig. S19). Next, we verified tailored crosslinking activity in response to varying transcriptional activation. In situ gelation kinetics were assessed after overnight growth in media containing a range of inducing molecule concentrations. Crosslinking activity was a strong function of IPTG concentration, spanning orders of magnitude in storage modulus (Fig. 4b). Crosslinking kinetics also corresponded to inducer presence, indicating that both synthesis rate and final material modulus can be customized through differential steady-state gene expression. Both an induced empty vector control and a complemented strain with no IPTG did not form measurable gels in $2 \mathrm{~h}$. Thus, transcriptional regulation over EET gene expression in response to an environmental signal imparts programmable control over hydrogel stiffness.

\section{Modeling Gene Expression Enables Predictable Material Properties}

Due to successful transcriptional regulation of $m t r C$, we hypothesized that a material property such as storage modulus could be predicted from inducible gene expression models. Since the sfgfp and $m t r C$ circuits have identical transcriptional regulation, we tested whether both fit to activating Hill function models. First, we measured the response function of the sfgfp circuit in S. oneidensis MR-1 by inducing overnight cultures in a variety of IPTG concentrations. Steadystate fluorescence was quantified using a plate reader, and normalized to optical density (Fig. 4c). As expected, relative expression (i.e., normalized fluorescence) was a strong function of IPTG concentration, and fit well to a Hill function with a hillslope of $n=1.57$ and a half-maximal effective concentration of $E C_{50}=98.6 \mu \mathrm{M}$ (Table S5). These results indicate that our circuit generates a predictable transcriptional response. Next, end-point gel measurements were used to examine storage modulus as a function of steady-state cytochrome (MtrC) expression. Gels were crosslinked for $2 \mathrm{~h}$ since our in situ results indicated this time would provide sufficient 298 differentiation between induced cultures at varying IPTG concentrations. Specifically, $299 \Delta m t r C \Delta o m c A \Delta m t r F$ complemented with Lacl-regulated $m t r C$ was grown overnight in a variety of 300 IPTG concentrations and allowed to react for $2 \mathrm{~h}$ at standard gelation conditions. We found that 
301 hydrogel storage moduli were also under strong transcriptional control, similar to sfgfp, and could

302 be modeled using a Hill function with $n=1.40$ and $E C_{50}=96.4 \mu \mathrm{M}$ (Fig. 4d). As the sfGFP signal

303 is effectively a measure of the transcriptional rate at different IPTG concentrations(43), the

304 similarity between fitted constants for sfgfp expression and hydrogel stiffness suggests a model

305 where transcriptionally-controlled MtrC levels predictably control hydrogel properties. To further

306 visualize this relationship, we plotted normalized storage modulus as a function of relative

307 expression units for each corresponding IPTG concentration and observed a linear correlation

308 (Fig. 4e). The 1:1 relationship between steady-state gene expression and hydrogel properties is

309 corroborated by the approximate unity of the slope. Together, these results demonstrate that EET

310 gene expression can be modulated to control ELM properties (e.g., gel stiffness), and that

311 fluorescence-parameterized models for existing and new genetic circuits may be adapted to

312 design, predict, and control more complex macroscopic material outputs.

313 Discussion:

We showed that $S$. oneidensis can genetically control radical crosslinking in a semisynthetic hydrogel via electron transfer to a redox-active polymerization catalyst. Similar to other crosslinking chemistries, storage modulus was dependent on catalyst and initiator concentrations as well as initial cell density. A significant advantage of our system is that it is theoretically amenable to any substrate that can undergo radical crosslinking and support microbial life. We used methacrylate-functionalized hyaluronic acid, but other semi-synthetic materials based on functionalized alginate, collagen, and cellulose, as well as completely synthetic substrates, such as PEG, should show similar behavior. In addition to flexibility in macromer structure, our design also allows for a variety of well-defined ATRP initiators to be used as crosslinking agents. For example, we showed two traditional ATRP initiators, HEBIB and bis-brominated PEG, could both form crosslinked hyaluronic acid hydrogels. Similar to other radical crosslinking methodologies, bacteria-controlled crosslinking is also compatible with various biochemical modifications including the installation of integrin recognition motifs (e.g., RGD), orthogonal crosslinking chemistries (e.g., Michael addition), and other common polymer engineering paradigms. The chemical flexibility and general compatibility with a variety of polymer network scaffolds should facilitate the use of our platform in tissue engineering, 3D printing, soft robotics, and drug delivery.

In contrast to other biologically-driven radical crosslinking methods, most notably hydroxyl radicals generated from glucose oxidase, EET-controlled crosslinking did not negatively impact cell health. Cells remained viable at least one week following gelation and transformed cells could 333 express sfgfp in response to an external stimulus. We also observed genotypic changes in cell 334 motility and convective flow as a result of EET-dependent crosslinking, implying genetic control 
over gel microstructure. Overall, our design avoids cell viability concerns associated with other radical crosslinking methodologies and could enable synthetic platforms for studying biofilm formation(44), trapping(45), or functionalizing cells(46).

We found that crosslinking activity and overall hydrogel stiffness were governed by EET cytochrome expression. Specifically, S. oneidensis MR-1 with wild-type EET pathways generated stiff gels within an hour while negative controls containing E. coli MG1655, which lacks EET machinery, did not form gels on comparable timescales. At longer time scales (ca. 3-4 hours), EET-knockout strains and E. coli showed some crosslinking activity. This background radical generation could be caused by non-specific copper reduction (e.g., release or secretion of cytosolic reducing agents) or spurious radical activation. To further reduce background crosslinking, decreasing catalyst and/or initiator concentration, lowering cell density, or changing the identity of chemical components could all potentially be tuned. Overall, the strong link between S. oneidensis genetics and crosslinking rate and density lays the foundation for developing more sophisticated EET-based regulation of material properties.

Using in situ and end-point rheology measurements, we showed that hydrogel crosslinking is directly linked to $m t r C$ expression levels, which has a number of implications for adaptable and dynamic materials. Interestingly, placing $m t r C$ under the control of the Lacl repressor generated a hydrogel stiffness response function that is characteristic of inducible gene expression. This response function mirrored one generated from sfgfp expression in the same construct, indicating robust transcriptional control over both gene expression and material properties. More importantly, our results suggest that previously characterized genetic circuits, including genetic logic gates, designed to express fluorescent reporters could be readily adapted to control $m t r C$ expression and gel stiffness(47). Although the ultimate stiffness of the hydrogel will depend on the specific crosslinking chemistry, our observation of canonical Hill function responses demonstrates that changes in hydrogel stiffness governed by transcriptional regulation can be partially predicted. Overall, our results suggest that a variety of transcriptional circuits could be extended to control the macroscopic properties of synthetic materials in a predictable and programmable manner. Additionally, robust genetic control over crosslinking should complement other stimuli-responsive hydrogel designs, including integration of biochemical signals(48-50), actuators $(51,52)$, and complex geometric designs(53).

The gel stiffness response function was measured after $2 \mathrm{~h}$ of gelation since in situ 366 measurements indicated this would be sufficient time to distinguish between differentially induced cells. Because crosslinking is a dynamic process, the hydrogel response function also varies as

368 a function of time, even at steady-state expression levels of MtrC. For example, at early times (< 
$1 \mathrm{~h}$ ), it is exaggerated since measurable gels do not form at low induction levels (Fig. S20). At longer timescales, the response function begins to collapse as background polymerization starts to compete with EET-driven crosslinking. In addition to understanding how EET influences crosslinking dynamics, many applications leveraging genetic control over material properties will likely require real-time transcriptional responses. Understanding how cells optimize transient gene expression to control dynamic outputs such as cell motility, morphogenesis, biofilm structure, or extracellular matrix construction are ongoing challenges in developmental and systems biology(54). Similarly, we are currently investigating how to coordinate transient gene expression to the polymerization kinetics in our system(55). Overall, continued optimization of MtrC (or other EET protein) expression and material chemistry should allow for actuation of material changes over timescales similar to transcription and translation, as well as predictive models that relate gene expression to material function.

Overall, we found that extracellular electron transfer from $S$. oneidensis could power a radical polymerization catalyst and form a semi-synthetic hydrogel composed of functionalized hyaluronic acid. A variety of chemical and biological factors controlled crosslinking kinetics and

384 the resulting storage moduli of the gels, demonstrating a tunable and adaptable platform. Most

385 importantly, we found that robust transcriptional control over $m t r C$ expression and metabolic 386 electron flux enabled precise and predictable control over hydrogel mechanical properties. While 387 cells are frequently incorporated into polymer networks, our platform allows for a variety of 388 network properties including crosslink density, mesh size, degradation, diffusion, and elastic 389 modulus to be controlled through cellular metabolism and gene expression. In summary, our results provide a powerful foundation to program adaptive and responsive behavior into the vast functional space of synthetic materials through the conduit of biological electron transfer.

Acknowledgements: S. oneidensis knockouts were a generous gift from Prof. Jeffrey Gralnick (U. Minnesota). A.J.G. was supported through a National Science Foundation Graduate Research Fellowship (Program Award No. DGE-1610403). This research was supported by the Welch Foundation (Grants F-1929), and by the National Science Foundation through the Center for Dynamics and Control of Materials: an NSF Materials Research Science and Engineering Center under DMR- 1720595. The authors acknowledge use of shared research facilities supported in part by the Texas Materials Institute, the Center for Dynamics and Control of Materials: an NSF MRSEC (DMR-1720595), and the NSF National Nanotechnology Coordinated Infrastructure 
402 (\#1015895) from the Burroughs Wellcome Fund. We gratefully acknowledge the use of facilities 403 within the core microscopy lab of the Institute for Cellular and Molecular Biology, University of 404 Texas at Austin. NMR spectra were collected on a Bruker Avance III 500 funded by the NIH 405 (Award 1 S10 OD021508-01) and a Bruker Avance III HD 400 funded by the NSF (Award CHE 406 1626211).

407

408 Correspondence: Correspondence should be addressed to keitz@utexas.edu and 409 arosales@che.utexas.edu.

411 Author Contributions: A.J.G., A.M.R., and B.K.K. conceived the project; A.J.G., C.M.D., and 412 D.S.K. performed experiments; C.M.D. designed and built the sfgfp and mtrC expression 413 plasmids; A.H. assisted with MeHA synthesis and rheology; A.J.G., C.M.D., A.M.R., and B.K.K. 414 analyzed the results; A.J.G., C.M.D., A.M.R., and B.K.K. wrote the manuscript.

415

\section{ORCID:}

417 Austin J. Graham: 0000-0001-8924-181X

418 Christopher M. Dundas: 0000-0001-9183-8236

419 Adrianne M. Rosales: 0000-0003-0207-7661

420 Benjamin K. Keitz: 0000-0003-3314-0053 


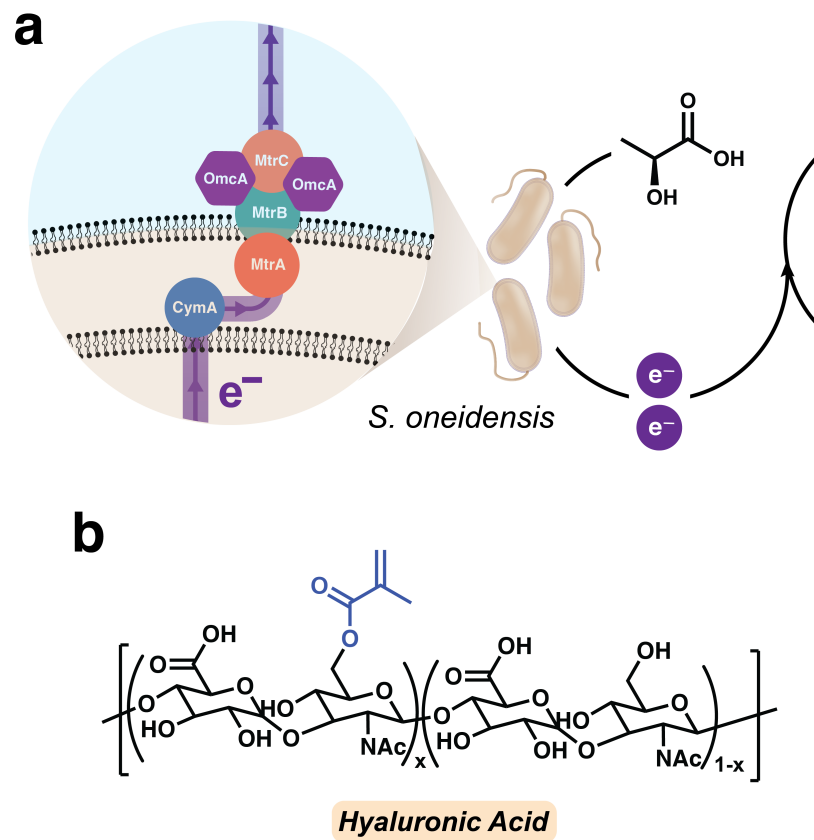

Hyaluronic Acid
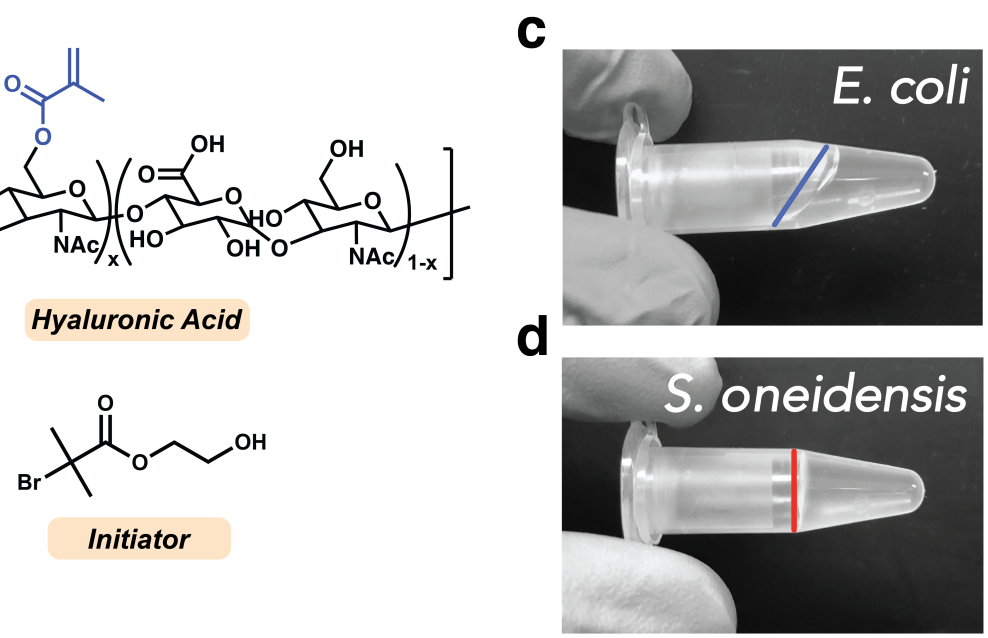

Figure 1. Extracellular electron transfer from $S$. oneidensis controls radical crosslinking of a semi-synthetic hydrogel. (a) The Mtr pathway of $S$. oneidensis transfers metabolic electron flux to a metal catalyst, which generates a radical from a brominated initiator and crosslinks acrylate-based functional groups. (b) Chemical structures of the macromer, methacrylated hyaluronic acid (MeHA), and the radical initiator, 2hydroxyethyl 2-bromoisobutyrate (HEBIB). (c) Crosslinking reaction mixture inoculated with $E$. coli, which does not possess EET machinery, does not form gels as indicated by liquid flow. Air-liquid interface is highlighted. (d) Crosslinking reaction mixture inoculated with $S$. oneidensis MR-1 forms a solid gel as confirmed by inversion test. Air-liquid interface is highlighted. 

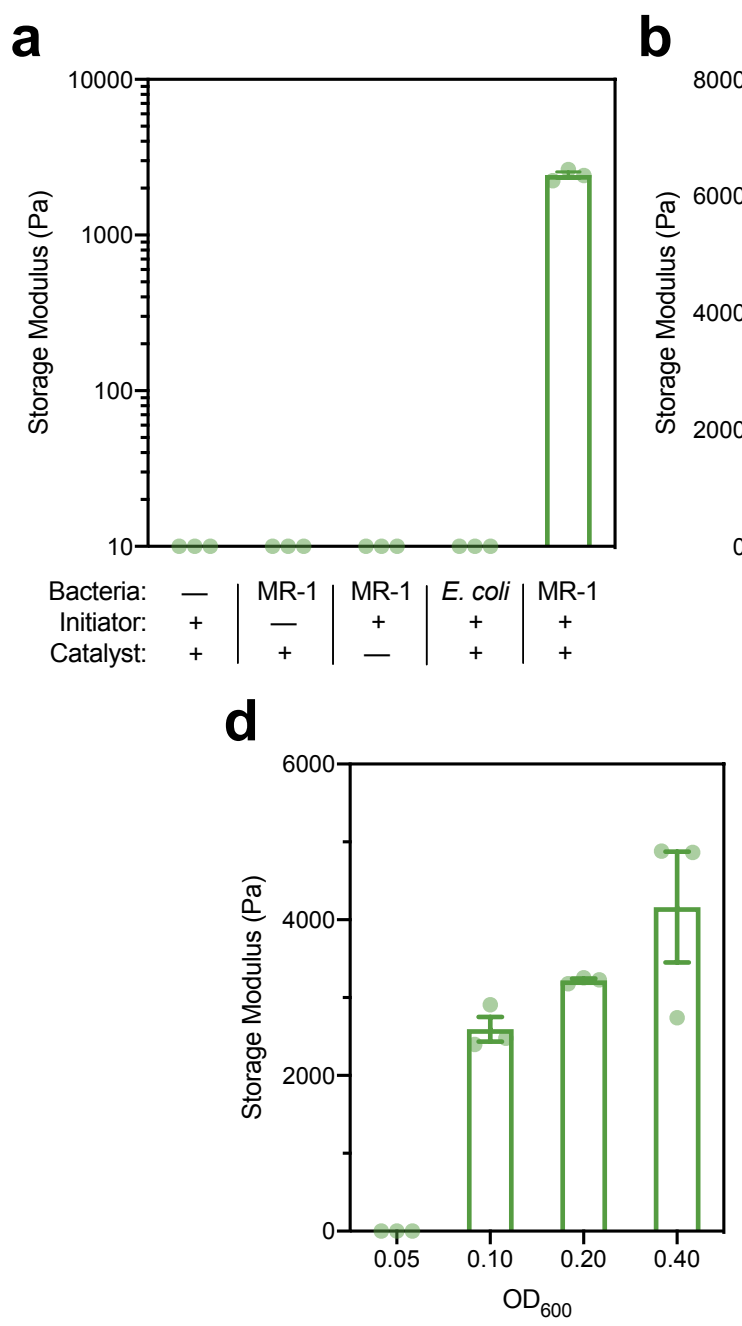

b



C

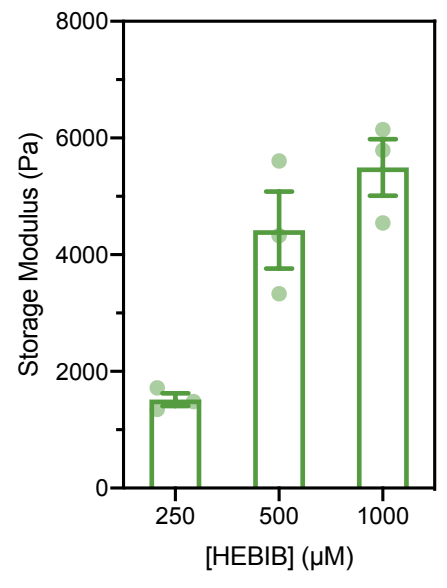

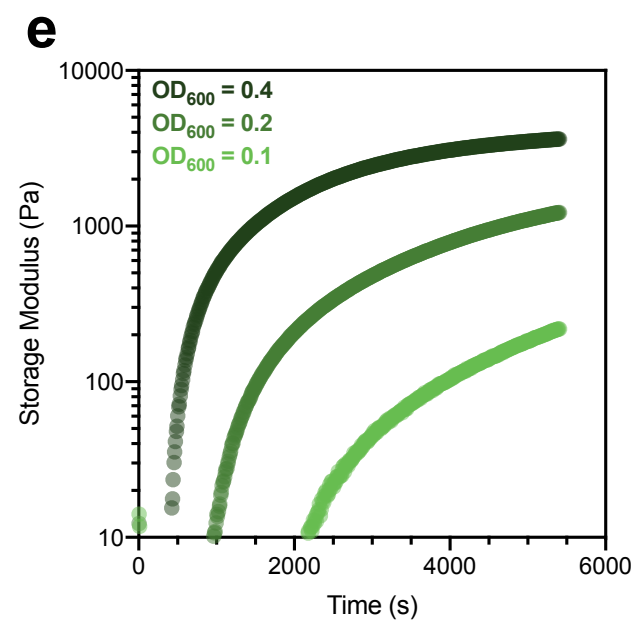

Figure 2. Living hydrogel materials crosslinked by S. oneidensis MR-1 can be chemically or biologically tuned. (a) Storage moduli of hydrogels crosslinked with various components missing measured by rheology after 2 hours of crosslinking. Many gels did not form and could not be characterized. (b) Storage moduli of hydrogels crosslinked for 2 hours with various concentrations of Cu-TPMA (catalyst), one-way ANOVA $p=$ 0.018. (c) Storage moduli of hydrogels crosslinked for 2 hours with various concentrations of HEBIB (radical initiator), one-way ANOVA $p=0.0027$. (d) End-point storage moduli at 2 hours and (e) in situ rheology of hydrogels crosslinked with various inoculating densities of $S$. oneidensis MR-1 and therefore varying degrees of electron transfer, one-way ANOVA $p=0.0002$. Gels did not form using $\mathrm{OD}_{600}=0.05$ in either experiment. (a-d) Data are shown as mean \pm SEM, $n=3$ biological replicates. 
bioRxiv preprint doi: https://doi.org/10.1101/752436; this version posted September 4 , 2019. The copyright holder for this preprint (which was not certified by peer review) is the author/funder, who has granted bioRxiv a license to display the preprint in perpetuity. It is made available under aCC-BY-NC-ND 4.0 International license.

a

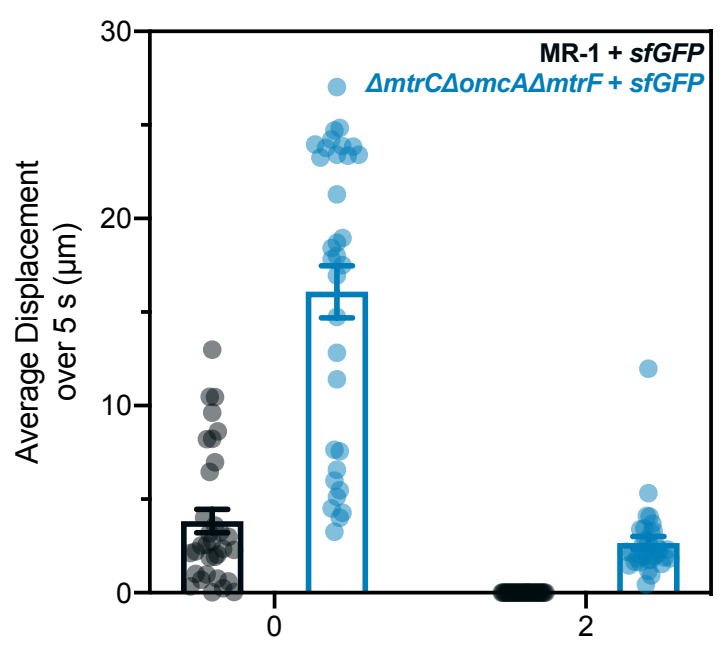

b

Reaction Time (h)
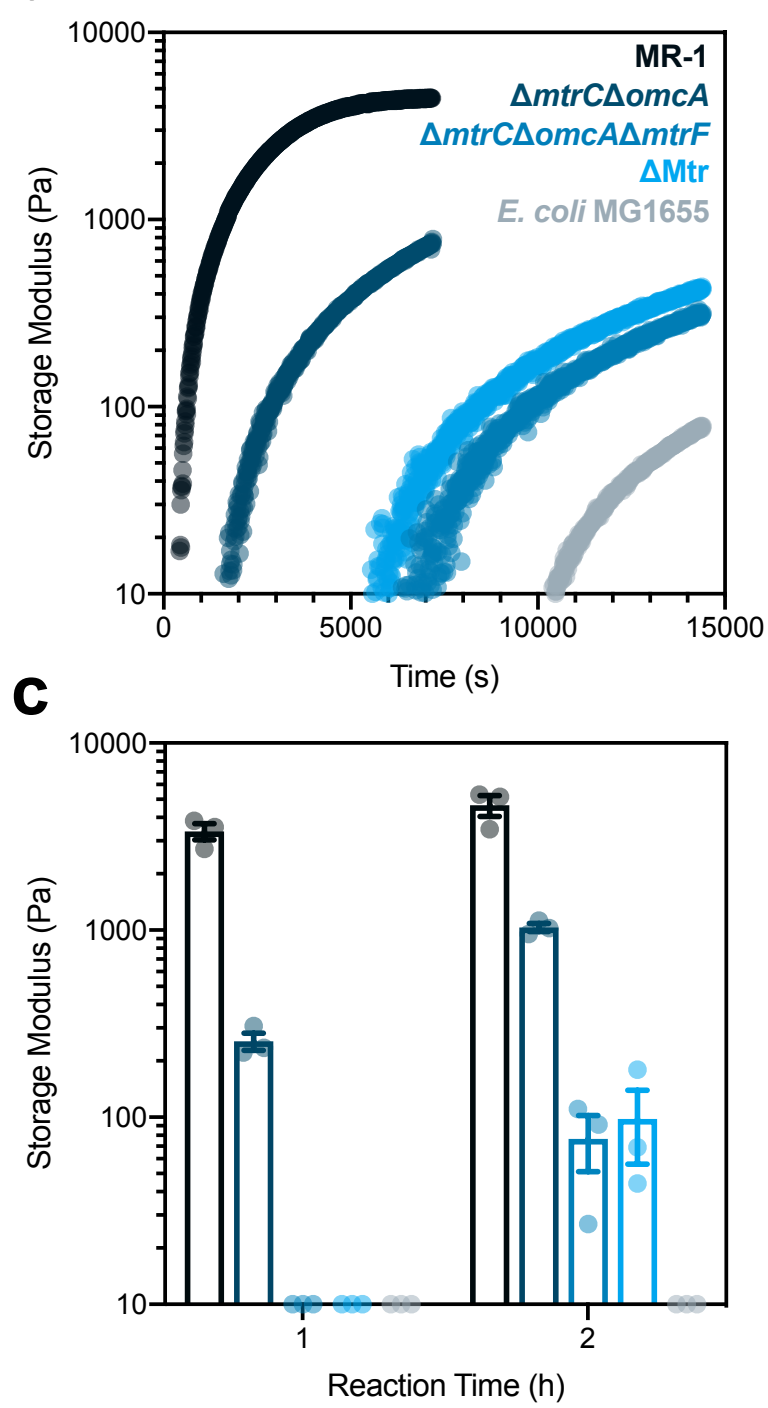
Figure 3. Crosslink density, synthesis rate, and cell motility within living hydrogels are governed by $S$. oneidensis genetics and EET machinery. (a) Average cell displacement within the gels measured by microscopy over 5 second time-lapses at both 0 and 2 hours into crosslinking. Displacement was quantified using TrackMate in Fiji 1.0. Student t-test $\mathrm{p}<0.0001$ between strains at $t=0$ and 2 hours. (b) In situ and (c) end-point rheology measurements of hydrogels crosslinked by S. oneidensis strains with various EET genes knocked out. E. coli was included as an EET-deficient control. Student t-test $p<0.0001$ for MR-1 compared to other strains at $t=1$ and 2 hours. Data are shown as mean $\pm \mathrm{SEM}$, (a) $n=33$ tracked cells or (c) $n=3$ biological replicates.

a
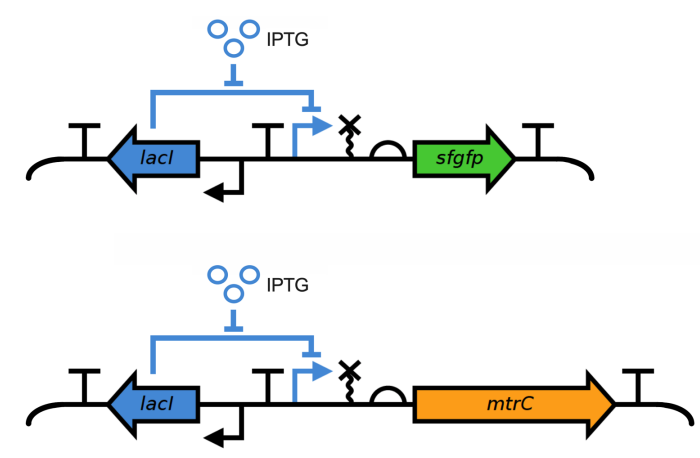

b

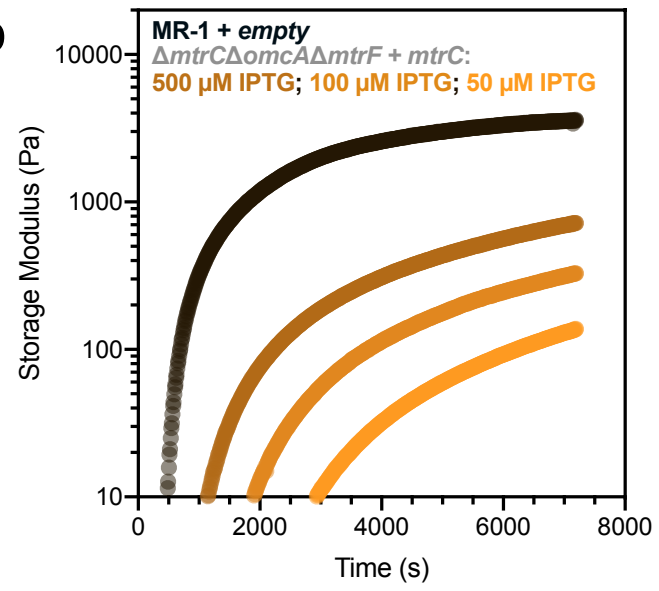

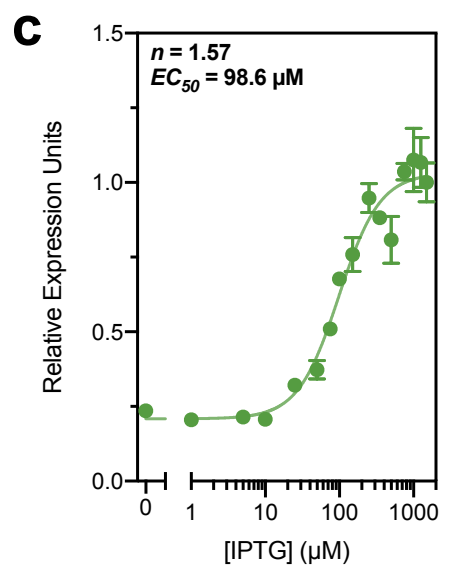
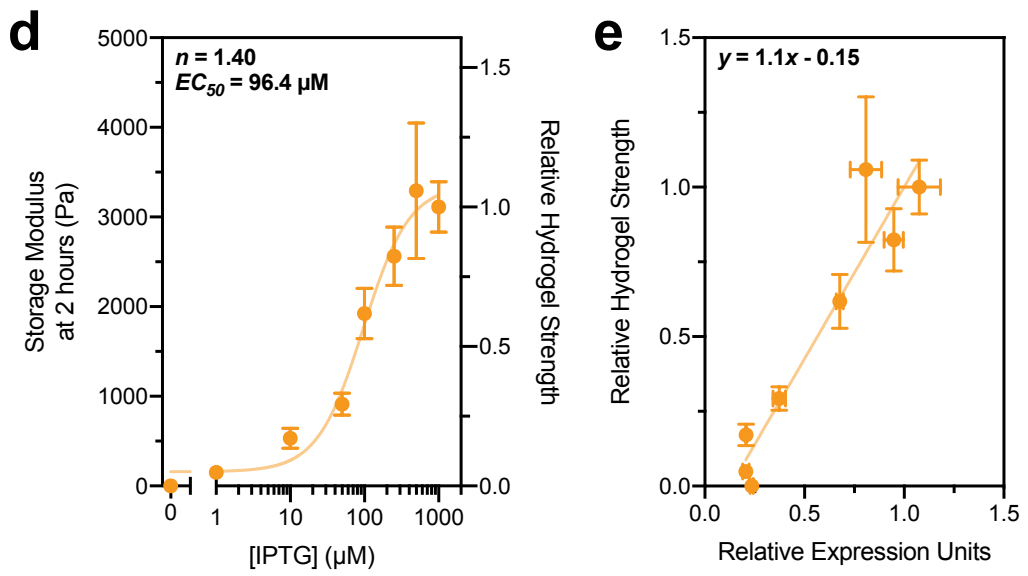

Figure 4. Modeling gene expression allows quantitative prediction of living hydrogel properties. (a) Genetic circuits utilized in this study placed either sfgfp or mtrC under inducible control of IPTG via the Lacl repressor and $\mathrm{P}_{\text {tac }}$ promoter. (b) In situ rheology of hydrogels crosslinked by $S$. oneidensis with steady-state induced $m t r C$ levels at various IPTG concentrations. $\Delta m t r C \Delta o m c A \Delta m t r F+$ empty and $\Delta m t r C \Delta o m c A \Delta m t r F$ $+m t r C$ with $0 \mu \mathrm{M}$ IPTG were also tested, but did not form gels on the time scale shown. (c) Hill function analysis of sfGFP fluorescence (denoted as relative expression units by normalization to fluorescence at maximum induction) as a function of IPTG concentration. (d) Hill function analysis of hydrogel storage modulus after 2 hours of crosslinking as a function of IPTG concentration. Right y-axis is storage modulus normalized to average modulus at maximum induction. (e) Normalized hydrogel stiffness plotted as a function of relative expression units for corresponding IPTG concentrations, fit to a line $\left(R^{2}=0.80\right)$. (c-e) Data are shown as mean \pm SEM, $n=3$ biological replicates. 


\section{Materials and Methods:}

\section{Chemicals and Reagents}

Sodium hyaluronate (72 kDa, Lifecore Biomedical), methacrylic anhydride (Sigma-Aldrich, 94\%), copper(II) bromide ( $\mathrm{CuBr}_{2}$, Sigma-Aldrich, 99\%), tris(2-pyridylmethyl)amine (TPMA, SigmaAldrich, 98\%), 2-hydroxyethyl 2-bromoisobutyrate (HEBIB, Sigma-Aldrich, 95\%), poly(ethylene glycol) bis(2-bromoisobutyrate) (PEGBBIB, $M_{n, a v g}=700 \mathrm{~g} / \mathrm{mol}$, Sigma-Aldrich, $\mathrm{PDI} \leq 1.1$ ) sodium DL-lactate $\left(\mathrm{NaC}_{3} \mathrm{H}_{5} \mathrm{O}_{3}, \mathrm{TCl}, 60 \%\right.$ in water), sodium fumarate $\left(\mathrm{Na}_{2} \mathrm{C}_{4} \mathrm{H}_{2} \mathrm{O}_{4}\right.$, VWR, $\left.98 \%\right)$, HEPES buffer solution $\left(\mathrm{C}_{8} \mathrm{H}_{18} \mathrm{~N}_{2} \mathrm{O}_{4} \mathrm{~S}, V W R, 1 \mathrm{M}\right.$ in water, $\left.\mathrm{pH}=7.3\right)$, potassium phosphate dibasic $\left(\mathrm{K}_{2} \mathrm{HPO}_{4}\right.$, Sigma-Aldrich), potassium phosphate monobasic $\left(\mathrm{KH}_{2} \mathrm{PO}_{4}\right.$, Sigma-Aldrich), sodium chlrodie (NaCl, VWR), ammonium sulfate $\left(\left(\mathrm{NH}_{4}\right)_{2} \mathrm{SO}_{4}\right.$, Fisher Scientific), magnesium (II) sulfate heptahydrate $\left(\mathrm{MgSO}_{4} \cdot 7 \mathrm{H}_{2} \mathrm{O}, \mathrm{VWR}\right)$, trace mineral supplement (ATCC), casamino acids (VWR), silicone oil (Alfa Aesar), isopropyl ß-D-1-thiogalactopyranoside (IPTG, Teknova), kanamycin sulfate $\left(\mathrm{C}_{18} \mathrm{H}_{38} \mathrm{~N}_{4} \mathrm{O}_{15} \mathrm{~S}\right.$, Growcells), nail polish (Electron Microscopy Sciences), BacLight Live/Dead Stain (Invitrogen), deuterium oxide $\left(\mathrm{D}_{2} \mathrm{O}\right.$, Sigma-Aldrich, $\left.99.9 \%\right)$, hydrogen peroxide solution $\left(\mathrm{H}_{2} \mathrm{O}_{2}\right.$, Sigma-Aldrich, $30 \%$ in $\mathrm{H}_{2} \mathrm{O}$ ), and 3,3',5,5'-Tetramethylbenzidine (TMBZ, Alfa Aesar, 98\%) were used as received. All media components were autoclaved or sterilized using $0.2 \mu \mathrm{m}$ PES filters.

\section{Methacrylated Hyaluronic Acid Synthesis and Purification}

MeHA was functionalized using methacrylic anhydride according to an established protocol(29). Briefly, $72 \mathrm{kDa}$ HA macromer $(1.5 \mathrm{~g}, 3.81 \mathrm{mmol}, 1.0 \mathrm{eq})$ was dissolved at $1 \mathrm{wt} \%$ in $\mathrm{DI}$ water $(150 \mathrm{~mL})$, cooled on ice, and adjusted to $\mathrm{pH}=8.5$ using $5 \mathrm{~N} \mathrm{NaOH}$. The $\mathrm{pH}$ was maintained between $7.5-8.5$ using $\mathrm{NaOH}$ while methacrylic anhydride $(8.44 \mathrm{~mL}, 56.7 \mathrm{mmol}$, $14.9 \mathrm{eq}$ ) was added in $750 \mu \mathrm{L}$ aliquots every $\sim 5$ minutes. Once all the methacrylic anhydride was added, the $\mathrm{pH}$ was maintained between $7.5-8.5$ for 4 hours, then the reaction stoppered and stirred overnight at room temperature. The reaction solution was dialyzed using $6-8 \mathrm{kDa}$ dialysis tubing in DI water while stirring for two weeks. The mixture was then frozen and lyophilized. Methacrylate functionalization was quantified by ${ }^{1} \mathrm{H}-\mathrm{NMR}$ spectroscopy and determined to be $\sim 65 \%$ from integration of the vinyl group relative to the HA backbone (Fig. S1). Functionalized MeHA solution was passed through an alumina column immediately prior to crosslinking reactions.

\section{Bacteria Strains and Culture}

Bacterial strains and plasmids are listed in Table S1 Cultures were prepared as follows: bacterial stocks stored in $20 \%$ glycerol at $-80^{\circ} \mathrm{C}$ were streaked onto LB agar plates (for wild-type and knockout strains) or LB agar with $25 \mu \mathrm{g} / \mathrm{mL}$ kanamycin (for plasmid-harboring strains) and grown overnight at $30^{\circ} \mathrm{C}$ for Shewanella, $37^{\circ} \mathrm{C}$ for $E$. coli. Single colonies were isolated and inoculated into Shewanella Basal Medium (SBM) supplemented with $100 \mathrm{mM} \mathrm{HEPES,} \mathrm{0.5 \%} \mathrm{trace}$ mineral supplement, $0.5 \%$ casamino acids, and $20 \mathrm{mM} 60 \%$ sodium lactate $(2.85 \mu \mathrm{L} / \mathrm{mL})$ as the electron donor. Aerobic cultures were pregrown in plastic $15 \mathrm{~mL}$ culture tubes at $30^{\circ} \mathrm{C}$ and 250 $\mathrm{rpm}$ shaking. Anaerobic cultures were pregrown using the same procedure outlined above, but in degassed medium in a humidified anaerobic chamber and supplemented with $40 \mathrm{mM}$ sodium fumarate $(40 \mu \mathrm{L} / \mathrm{mL}$ of a $1 \mathrm{M}$ stock) as the electron acceptor. Cultures were washed after pregrowth using SBM supplemented with $0.5 \%$ casamino acids (degassed for anaerobic cultures). $\mathrm{OD}_{600}$ was measured using a NanoDrop 2000C spectrophotometer and normalized to 2.0 for 10 -fold dilution into gel mixtures unless otherwise noted.

\section{MeHA Hydrogel Crosslinking using S. oneidensis}

$\mathrm{CuBr}_{2}, \mathrm{TPMA}$, and HEBIB stock solutions were prepared according to previously established protocol(21). For three $50 \mu \mathrm{L}$ hydrogel discs that were analyzed by rheology, a 
reaction mixture was prepared as follows: MeHA was dissolved at $3.76 \mathrm{wt} \%$ in SBM with $0.5 \%$ casamino acids and aliquoted into an autoclaved microfuge tube (119.2 $\mu \mathrm{L}) .400 \mu \mathrm{M} \mathrm{Cu}$-TPMA $(3.75 \mu \mathrm{L}), 69 \mathrm{mM}$ HEBIB $(1.09 \mu \mathrm{L}), 60 \%$ sodium lactate $(0.428 \mu \mathrm{L})$, and $1 \mathrm{M}$ sodium fumarate $(6$ $\mu \mathrm{L})$ were added to the MeHA solution and mixed. Final concentrations in solution were 3 wt. $\%$ MeHA, $10 \mu \mathrm{M}$ Cu-TPMA, $500 \mu \mathrm{M}$ HEBIB, $20 \mathrm{mM}$ lactate, and $40 \mathrm{mM}$ fumarate. This solution was distributed into three autoclaved microfuge tubes of $45 \mu \mathrm{L}$ aliquots to which $5 \mu \mathrm{L}$ of $\mathrm{OD}_{600}$ normalized cells were added. The gel solutions were mixed and added to hydrophobically-treated glass slides with a $0.5 \mathrm{~mm}$ silicone spacer separating the two glass layers. Slides were sealed with a binder clip and allowed to react at $30^{\circ} \mathrm{C}$ for two hours unless otherwise noted. Hydrogels were removed from the slides using a razor blade and placed into $3 \mathrm{~mL}$ baths of 1x PBS overnight to swell to equilibrium. Hydrogels analyzed by in situ rheology were prepared using the same mixture outlined above, but inoculated with cells and immediately placed on the rheometer for analysis.

\section{Rheological Analysis}

End-point rheological analysis: swollen hydrogels prepared as outlined above were analyzed by oscillatory shear rheology using a TA Instruments Discovery HR-2 Rheometer with an $8 \mathrm{~mm}$ parallel plate geometry. Hydrogels were loaded onto a Peltier plate and excised to $8 \mathrm{~mm}$ diameter using a biopsy punch. The geometry gap was then lowered until the measured axial force was above $0.02 \mathrm{~N}$ (usually between $500-800 \mu \mathrm{m}$, depending on the crosslink density and swelling ratio). Storage and loss moduli were measured using frequency sweeps from 0.01 to 100 $\mathrm{Hz}$ at a constant strain of $0.1 \%$. Moduli for a single gel were quantified by averaging the linear viscoelastic region of each frequency sweep.

In situ rheological analysis: hydrogels measured by in situ oscillatory shear rheology were prepared using the mixtures and rheometer outlined above. Immediately after inoculating reaction mixtures with cells, $80 \mu \mathrm{L}$ of mix was loaded onto the Peltier plate, which was maintained at 30 ${ }^{\circ} \mathrm{C}$. A $20 \mathrm{~mm}$ parallel plate geometry was lowered onto the solution while spinning such that the mixture coated the entire geometry surface and filled the gap ( $350 \mu \mathrm{m}$ gap size). The edges of the geometry and gap were then coated with silicone oil to prevent evaporative losses. In situ crosslinking was monitored using $1 \mathrm{~Hz}$ oscillation and $0.1 \%$ strain over variable lengths of time $(1.5-8$ hours).

\section{Microscopy and Cell Tracking}

All microscopy was performed using a Nikon Ti2 Eclipse inverted fluorescence microscope. Cells assessed for viability by microscopy were crosslinked using standard conditions and the resulting gels swollen in 1x PBS at room temperature for varying lengths of time. The gels were then incubated in the dark in the BacLight Live/Dead stain mix $(1.5 \mu \mathrm{L} / \mathrm{mL}$ Syto9, $2.5 \mu \mathrm{L} / \mathrm{mL}$ propidium iodide in $0.85 \% \mathrm{NaCl}$ solution) for 30 minutes. Stained gels were then washed by pipetting $3 x$ in $1 \mathrm{~mL}$ PBS to remove unbound dye. Gels were loaded onto glass microscope slides and a no. 1 coverslip placed on top. The gel thickness prevented using nail polish to seal the sides, but evaporative losses were not noticeable over the course of the experiment. Fluorescence for each stain (green for Syto9, red for propidium iodide) was measured using GFP and Texas Red excitation/emission filter cubes on a Nikon Ti2 Eclipse, as outlined previously(21). To assess metabolic activity, gels were crosslinked with sfgfp-harboring strains and allowed to swell in 1x PBS for varying lengths of time. sfGFP fluorescence was assessed before induction to ensure there was no detectable background fluorescence. Gels were then incubated in $1000 \mu \mathrm{M}$ IPTG in PBS for 24 hours and monitored by fluorescence using the GFP channel.

Cells tracked by microscopy during crosslinking were prepared with reaction mixtures as outlined above. Upon cell inoculation, the crosslinking mixture was loaded onto glass slides, covered, and sealed with nail polish. The slides were loaded onto the microscope and cell 
movement monitored using the Time-lapse function in NIS-Elements. Images were taken every 1 $\mathrm{s}$ or $5 \mathrm{~s}$ with 100 or $300 \mathrm{~ms}$ exposure time using the GFP channel. Time-lapse images were edited and quantified using TrackMate in Fiji 1.0. Images were first background subtracted and equally brightened by thresholding. The top 50 highest quality cells were selected, as determined by the TrackMate user interface, and tracked over 5 or $10 \mathrm{~s}$. The highest quality tracks, as determined by the software, were used to quantify average total displacement over the time-lapse. The number of tracks used to calculate the average was the maximum number of tracks for the image with the fewest tracks at each time point.

\section{Plasmid Construction}

DNA sequences and plasmid maps for each genetic part and plasmid used in this study are given in the Supplementary Information. All plasmids were assembled via Golden Gate cloning using enzymes and buffers from New England Biolabs. In addition to T4 Ligase, Golden Gate reactions contained either Sapl for mtrC plasmid assembly or Bsal for sfgfp and empty plasmid assembly. The PCD backbone was assembled by PCR amplifying (Phusion, New England Biolabs) regions of pSR58.6 (B0015 and T0 terminators, ColE1 origin of replication), pTKEI-tLOV (kanamycin resistance), and pAL-rfp_(RP4 origin of transfer). To construct the gene expression unit (insulating terminators, RiboJ ribozyme, and lacl regulation unit), a gBlock was synthesized (Integrated DNA Technologies) and used in Golden Gate cloning. sfgfp and mtrC were PCR amplified from pSR58.6 and purified Shewanella oneidensis MR-1 genomic DNA, respectively, with ribosome binding sites and Golden Gate restriction enzyme sites added via oligonucleotide primers. Generally, $10 \mu \mathrm{L}$ Golden Gate reactions were set up that contained 10 fmol of pCD plasmid backbone and $40 \mathrm{fmol}$ of each gBlock and/or PCR insert (as necessary). In a thermocycler, Golden Gate reactions were cycled 25 times: $90 \mathrm{~s}$ at $37^{\circ} \mathrm{C}$ followed by 3 minutes at $16{ }^{\circ} \mathrm{C}$. After the 25 cycles, reactions were incubated at $37{ }^{\circ} \mathrm{C}$ for 5 minutes, $80{ }^{\circ} \mathrm{C}$ for 10 minutes, and then held at $4{ }^{\circ} \mathrm{C}$.

Golden Gate reactions were used to directly transform freshly prepared electrocompetent S. oneidensis strains(56). To prepare electrocompetent $S$. oneidensis, $5 \mathrm{~mL}$ of overnight $S$. oneidensis growth in LB medium at $30{ }^{\circ} \mathrm{C}$ was washed 3 times with sterile $10 \%$ glycerol at room temperature and concentrated to $\sim 300 \mu \mathrm{L} .2 \mu \mathrm{L}$ of Golden Gate reaction was mixed with $30 \mu \mathrm{L}$ of concentrated electrocompetent $S$. oneidensis, transferred to a $1 \mathrm{~mm}$ electroporation cuvette, and electroporated at $1250 \mathrm{~V}$. To recover electroporated cells, $250 \mu \mathrm{L}$ of LB was immediately added post-electroporation and cells were incubated/shaken at $30^{\circ} \mathrm{C}$ and $250 \mathrm{rpm}$. After $2 \mathrm{~h}$ of recovery, $100 \mu \mathrm{L}$ of cell suspension was plated onto LB agar plates containing $25 \mu \mathrm{g} \mathrm{m}^{-1}$ kanamycin sulfate and incubated overnight at $30{ }^{\circ} \mathrm{C}$ to obtain single colonies (generally 5-100 colonies observered for 1-3 part assemblies). Single colonies were used to inoculate LB liquid medium containing 25 $\mu \mathrm{g} \mathrm{mL}{ }^{-1}$ kanamycin sulfate and incubated/shaken overnight at $30^{\circ} \mathrm{C}$ and $250 \mathrm{rpm}$. These cultures were used to generate $22.5 \%$ glycerol stocks, which were stored at $-80{ }^{\circ} \mathrm{C}$, and harvest assembled plasmid for Sanger sequencing (DNA Sequencing Facilities, University of Texas at Austin).

\section{Verification of MtrC Inducible Expression and Functional Activity}

Heme staining was performed by adapting previously described methods (57). $5 \mathrm{~mL}$ of uninduced (0 $\mu \mathrm{M}$ IPTG) and induced (1000 $\mu \mathrm{M}$ IPTG) S. oneidensis strains were anaerobically cultured overnight in SBM containing $20 \mathrm{mM}$ lactate and $40 \mathrm{mM}$ fumarate. The total culture was washed once in $1 \times$ PBS, concentrated to $500 \mu \mathrm{L}$, and lysed by sonication. The cell lysate was centrifuged for 10 minutes at 10,000 rcf, and the supernatant was transferred to a separate tube. The pellet was resuspended in $100 \mu \mathrm{L} 1 \mathrm{x}$ PBS, and the total protein concentration of both lysate fractions was determined by Bradford assay. $10 \mu \mathrm{g}$ of protein from the supernatant and pellet were loaded into each well of a 12\% Bis-Tris SDS-PAGE gel and run for ca. 120 minutes at 110 V. The gel was stained in a 3:7 mixture of $6.3 \mathrm{mM} \mathrm{TMBZ}$ in methanol:0.25 M sodium acetate $(\mathrm{pH}$ 
5.0) for $2 \mathrm{~h}$ in the dark. Heme-containing protein bands were visualized upon addition of $30 \mathrm{mM}$ hydrogen peroxide for $30 \mathrm{~min}$.

Ferrozine assay(58) was performed to determine functional activity of MtrC-expressing strains. Strains were anaerobically grown overnight in IPTG-free SBM containing $20 \mathrm{mM}$ lactate and $40 \mathrm{mM}$ fumarate. Within in anaerobic chamber, cells were diluted 100-fold into 96-well plate wells filled SBM containing $20 \mathrm{mM}$ lactate, $5 \mathrm{mM} \mathrm{Fe}$ (III)-citrate, and $1 \mathrm{mg} \mathrm{mL}^{-1}$ ferrozine (final total volume of $250 \mu \mathrm{L}$ per well). Each well contained either 0 or $750 \mu \mathrm{M}$ IPTG and Fe(II) standards were also added to the plate. Immediately after addition of cells and $\mathrm{Fe}(\mathrm{III})$, the plate was sealed with optically transparent sealing film and a plate cover lined with silicone grease. The plate was then removed from the anaerobic chamber and statically incubated at $30{ }^{\circ} \mathrm{C}$. Absorbance at 562 $\mathrm{nm}$ was periodically measured using a BMG LABTECH CLARIOstar plate reader.

\section{Quantification and Modeling of Inducible Constructs}

The $\Delta m t r C \Delta o m c A \Delta m t r F+s f g f p$ and $+m t r C$ strains were anaerobically pregrown overnight using the same conditions outlined above, with the addition of $25 \mu \mathrm{g} \mathrm{mL}^{-1}$ kanamycin and varying IPTG concentrations. Cells were anaerobically washed $3 x$ in degassed SBM with $0.05 \%$ casamino acids, normalized to $\mathrm{OD}_{600}=2.0$, and diluted 10 -fold into reaction mixtures (also containing kanamycin and IPTG) prepared in ambient conditions. Prior to measuring sfGFP fluorescence, protein translation was arrested by supplementing a $100 \mu \mathrm{L}$ aliquot of cell suspension with kanamycin sulfate to a final concentration of $2 \mathrm{mg} \mathrm{mL}^{-1}$. Subsequently, this suspension was shaken aerobically for $1 \mathrm{~h}$ at $30{ }^{\circ} \mathrm{C}$ to allow for sfGFP maturation. sfGFP fluorescence $(488 / 530 \mathrm{~nm})$ and cell suspension absorbance $(600 \mathrm{~nm})$ was measured using a BMG LABTECH CLARIOstar plate reader to yield fluorescence absorbance ${ }^{-1}$ for each sample. For each sample, the background fluorescence absorbance ${ }^{-1}$ from an empty vector ( $\left.p C D 8\right)$ control was subtracted. The background subtracted values were then normalized to the average fluorescence absorbance ${ }^{-1}$ value at maximum induction $(1500 \mu \mathrm{M}$ IPTG) to give relative expression units. Crosslinking strains complemented with $m$ tr $C$ were allowed to form gels for two hours, and the gels allowed to swell overnight in 1x PBS at room temperature. Gels were then analyzed by oscillatory shear rheology as outlined above. A nonlinear fitting algorithm in GraphPad Prism 8.0 was used to fit inducible gene expression and hydrogel storage modulus to the following activating Hill function: $y=$ Bottom $+($ Top - Bottom $) \frac{[I]^{n}}{E C_{50}{ }^{n}+[]^{n}}$. Normalized hydrogel storage modulus and relative expression units were plotted for values at corresponding IPTG concentrations and modeled using a linear fit. Further details on modeling can be found in Note S1. Fitting parameters and "goodness of fit" can be found in Table S5.

\section{Statistical Analysis}

Unless otherwise noted, data are reported as mean \pm SEM of $n=3$ biological replicates. Significance was calculated in GraphPad Prism 8.0 using either a two-tailed unpaired student ttest or a one-way ANOVA $(\alpha=0.05)$.

\section{Data deposition}

Experimental data supporting the findings in this study are publicly available through the Texas Data Repository (doi: XXX).

\section{References:}


2. Chen AY, Zhong C, Lu TK (2014) Engineering Living Functional Materials. ACS Synthetic Biology 4(1):8-11.

3. Gilbert C, Ellis T (2019) Biological Engineered Living Materials: Growing Functional Materials with Genetically Programmable Properties. ACS Synthetic Biology 8(1):115.

4. Nguyen PQ, Courchesne N-MD, Duraj-Thatte A, Praveschotinunt $P$, Joshi NS (2018) Engineered Living Materials: Prospects and Challenges for Using Biological Systems to Direct the Assembly of Smart Materials. Adv Mater 30(19):1704847-34.

5. Chen AY, et al. (2014) Synthesis and patterning of tunable multiscale materials with engineered cells. Nature Materials 13(5):515-523.

6. Appiah C, et al. (2019) Living Materials Herald a New Era in Soft Robotics. Adv Mater 9:1807747-28.

7. Shaffner M, Rühs PA, Coulter F, Kilcher S, Studart AR (2017) 3D printing of bacteria into functional complex materials. Sci Adv 3(12):eaao6804.

8. Liu X, et al. (2017) 3D Printing of Living Responsive Materials and Devices. Adv Mater 34:1704821-14.

9. Liu X, et al. (2017) Stretchable living materials and devices with hydrogel-elastomer hybrids hosting programmed cells. Proc Natl Acad Sci USA 114(9):2200-2205.

10. Mangayil R, et al. (2017) Engineering and Characterization of Bacterial Nanocellulose Films as Low Cost and Flexible Sensor Material. ACS Applied Materials \& Interfaces 9(22):19048-19056.

11. Nguyen PQ, Botyanszki Z, Tay PKR, Joshi NS (2014) Programmable biofilm-based materials from engineered curli nanofibres. Nature Communications 5:4945.

12. Kalyoncu E, Ahan RE, Ozcelik CE, Seker UOS (2019) Genetic Logic Gates Enable Patterning of Amyloid Nanofibers. Adv Mater.1902888-7.

13. Huang J, et al. (2018) Programmable and printable Bacillus subtilis biofilms as engineered living materials. Nat Chem Biol 15:34-41.

14. Botyanszki Z, Tay PKR, Nguyen PQ, Nussbaumer MG, Joshi NS (2015) Engineered catalytic biofilms: Site-specific enzyme immobilization onto $\mathrm{E}$. coli curli nanofibers. Biotechnol Bioeng 112(10):1-10.

15. Tay PKR, Nguyen PQ, Joshi NS (2017) A Synthetic Circuit for Mercury Bioremediation Using Self-Assembling Functional Amyloids. ACS Synthetic Biology 6(10):1841-1850.

16. Dorval Courchesne N-M, et al. (2018) Biomimetic engineering of conductive curli protein films. Nanotechnology 29(45):454002-13.

17. Duraj Thatte AM, et al. (2019) Genetically Programmable Self-Regenerating 
Bacterial Hydrogels. Adv Mater 3:1901826-9.

18. Thongsomboon W, et al. (2018) Phosphoethanolamine cellulose: A naturally produced chemically modified cellulose. Science 359: 334-338.

19. Florea M, et al. (2016) Engineering control of bacterial cellulose production using a genetic toolkit and a new cellulose-producing strain. Proc Natl Acad Sci USA 113(24):E3431-40.

20. McEvoy MA, Correll N (2015) Materials that couple sensing, actuation, computation, and communication. Science 347(6228):1261689-1261689.

21. Fan G, Dundas CM, Graham AJ, Lynd NA, Keitz BK (2018) Shewanella oneidensisas a living electrode for controlled radical polymerization. Proc Natl Acad Sci USA 4:201800869-6.

22. Gao H, Chan N, Oh JK, Matyjaszewski K (2015) Designing Hydrogels by ATRP. InSitu Gelling Polymers: for Biomedical Applications. ed Loh XJ (Springer Singapore, Singapore), pp 69-105.

23. Lee KY, Kong HJ, Larson RG, Mooney DJ (2003) Hydrogel Formation via Cell Crosslinking. Adv Mater 15(21):1828-1832.

24. Pitzler C, et al. (2014) A Fluorescent Hydrogel-Based Flow Cytometry HighThroughput Screening Platform for Hydrolytic Enzymes. Chemistry \& Biology 21(12):1733-1742.

25. Isdorf NLX, et al. (2015) A flow cytometer-based whole cell screening toolbox for directed hydrolase evolution through fluorescent hydrogels. Chemical Communications 51:8679-8682.

26. Nagahama K, Kimura Y, Takemoto A (2018) Living functional hydrogels generated by bioorthogonal cross-linking reactions of azide-modified cells with alkyne-modified polymers. Nature Communications 9:2195.

27. Liu Y, Sakai S, Kawa S, Taya M (2014) Identification of Hydrogen PeroxideSecreting Cells by Cytocompatible Coating with a Hydrogel Membrane. Anal Chem 86(23):11592-11598.

28. Huang A, et al. (2018) BioBitsTM Explorer: A modular synthetic biology education kit. Sci Adv 4:eeat5105.

29. Rodell CB, et al. (2014) Shear-Thinning Supramolecular Hydrogels with Secondary Autonomous Covalent Crosslinking to Modulate Viscoelastic Properties In Vivo. Adv Func Mater 25(4):636-644.

30. Xu X, Jha AK, Harrington DA, Farach-Carson MC, Jia X (2012) Hyaluronic acidbased hydrogels: from a natural polysaccharide to complex networks. Soft Matter 8(12):3280-15.

31. Matyjaszewski K (2012) Atom Transfer Radical Polymerization (ATRP): Current 
Status and Future Perspectives. Macromolecules 45(10):4015-4039.

32. Toes ACM, Daleke MH, Kuenen JG, Muyzer G (2008) Expression of copA and cusA in Shewanella during copper stress. Microbiology 154(9):2709-2718.

33. Böl M, Ehret AE, Bolea Albero A, Hellriegel J, Krull R (2012) Recent advances in mechanical characterisation of biofilm and their significance for material modelling. Critical Reviews in Biotechnology 33(2):145-171.

34. Engler AJ, Sen S, Sweeney HL, Discher DE (2006) Matrix Elasticity Directs Stem Cell Lineage Specification. Cell 126(4):677-689.

35. Carr DA, Peppas NA (2009) Molecular Structure of Physiologically-Responsive Hydrogels Controls Diffusive Behavior. Macromol Biosci 9(5):497-505.

36. Bathe M, Rutledge GC, Grodzinsky AJ, Tidor B (2005) A Coarse-Grained Molecular Model for Glycosaminoglycans: Application to Chondroitin, Chondroitin Sulfate, and Hyaluronic Acid. Biophysical Journal 88(6):3870-3887.

37. Paulick A, et al. (2009) Two different stator systems drive a single polar flagellum in Shewanella oneidensisMR-1. Molecular Microbiology 71(4):836-850.

38. Tinevez J-Y, et al. (2017) TrackMate: An open and extensible platform for singleparticle tracking. Methods 115:80-90.

39. Coursolle D, Gralnick JA (2010) Modularity of the Mtr respiratory pathway of Shewanella oneidensis strain MR-1. Molecular Microbiology 55:995-1008.

40. Clarke TA, et al. (2011) Structure of a bacterial cell surface decaheme electron conduit. Proc Natl Acad Sci USA 108:9384-9389.

41. Coursolle D, Gralnick JA (2012) Reconstruction of Extracellular Respiratory Pathways for Iron(III) Reduction in Shewanella Oneidensis Strain MR-1. Frontiers in Microbiology 3:1-11.

42. Farasat I, et al. (2014) Efficient search, mapping, and optimization of multi-protein genetic systems in diverse bacteria. Mol Syst Biol 10(6):731-18.

43. Kelly JR, et al. (2009) Measuring the activity of BioBrick promoters using an in vivo reference standard. Journal of Biological Engineering 3(1):4-13.

44. Zhang Y, Ng CK, Cohen Y, Cao B (2014) Cell growth and protein expression of Shewanella oneidensis in biofilms and hydrogel-entrapped cultures. Mol BioSyst 10(5):1035-8.

45. Park JH, Hong D, Lee J, Choi IS (2016) Cell-in-Shell Hybrids: Chemical Nanoencapsulation of Individual Cells. Accounts of Chemical Research 49(5):792800.

46. Niu J, et al. (2017) Engineering live cell surfaces with functional polymers via cytocompatible controlled radical polymerization. Nature Chemistry 9(6):537-545. 
47. Brophy JAN, Voigt CA (2014) Principles of genetic circuit design. Nature Methods 11: 508-520.

48. Lu Y, Aimetti AA, Langer R, Gu Z (2016) Bioresponsive materials. Nat Rev Mater 2(1):16075-17.

49. Badeau BA, Comerford MP, Arakawa CK, Shadish JA, DeForest CA (2018)

Engineered modular biomaterial logic gates for environmentally triggered therapeutic delivery. Nature Chemistry 10:251-258.

50. Wang $\mathrm{H}$, et al. (2017) Covalently Adaptable Elastin-Like Protein-Hyaluronic Acid (ELP-HA) Hybrid Hydrogels with Secondary Thermoresponsive Crosslinking for Injectable Stem Cell Delivery. Adv Func Mater 27(28):1605609-11.

51. Ionov L (2014) Hydrogel-based actuators: possibilities and limitations. Biochemical Pharmacology 17(10):494-503.

52. de Almeida P, et al. (2019) Cytoskeletal stiffening in synthetic hydrogels. Nature Communications 10:609.

53. $\quad \mathrm{Na} \mathrm{J}-\mathrm{H}$, et al. (2014) Programming Reversibly Self-Folding Origami with Micropatterned Photo-Crosslinkable Polymer Trilayers. Adv Mater 27(1):79-85.

54. Yosef N, Regev A (2011) Impulse Control: Temporal Dynamics in Gene Transcription. Cell 144(6):886-896.

55. Ang J, Harris E, Hussey BJ, Kil R, McMillen DR (2013) Tuning Response Curves for Synthetic Biology. ACS Synthetic Biology 2(10):547-567.

56. Corts AD, Thomason LC, Gill RT, Gralnick JA (2019) A new recombineering system for precise genome-editing in Shewanella oneidensis strain MR-1 using singlestranded oligonucleotides. Scientific Reports 9:39.

57. Bradford MM (1976) A Rapid and Sensitive Method for the Quantitation of Microgram Quantities of Protein Utilizing the Principle of Protein-Binding Dye. Analytical Biochemistry 72:248-254.

58. Stookey LL (1970) Ferrozine-A New Spectrophotometric Reagent for Iron. Analytical Chemistry 42(7):779-781. 